\title{
Characterization of Tumor Imaging with Microbubble-Based Ultrasound Contrast Agent, Sonazoid, in Rabbit Liver
}

\author{
Rira Watanabe,* Manabu Matsumura, Chun-Jen Chen, Yuki Kaneda, and Masayoshi Fujimaki \\ New Product Research Laboratories II, Daiichi Pharmaceutical Co., Ltd.; 1-16-13 Kita-kasai, Edogawa-ku, Tokyo \\ 134-8630, Japan. $\quad$ Received December 27, 2004; accepted April 4, 2005; published online April 6, 2005
}

\begin{abstract}
In order to investigate improvement of hepatic tumor detectability by Sonazoid with phase inversion imaging, the contrast effects on the liver of metastatic carcinoma-model rabbits were evaluated by videodensitometry and visual assessment. Correlation between the contrast enhancement of Sonazoid and histopathology was examined using the same animals. Electron microscopy was performed on hepatic tissue from another healthy rabbits to identify the distribution of Sonazoid microbubbles. As a result, all tumors were smaller than $\mathbf{1 2} \mathbf{~ m m}$ in diameter, and after intravenous injection of Sonazoid, they were surrounded with a ring of enhanced signal during the early phase (up to $30 \mathrm{~s}$ ), followed by a clear contrast defect during the delayed phase (after $10 \mathrm{~min}$ ). Histopathologic observation revealed that the ring-enhancement was caused by neovasculature in the tumor, and the contrast defects corresponded to living and dead parts of tumors, which lack Kupffer cells. Videodensitometric differences between tumor and healthy tissue markedly increased at delayed phase, and visual detectability of tumors was improved when Sonazoid was used. Ultrastructural analysis showed microbubble-like structures in Kupffer cells, which indicated that Sonazoid microbubbles were taken up with these cells. In conclusion, Sonazoid, used with phase inversion imaging, greatly increases the detectability of small hepatic tumors by highlighting neovascularity at early phase and providing clear contrast defects due to absence of Kupffer cells, which take up Sonazoid microbubbles, at delayed phase.
\end{abstract}

Key words ultrasound contrast agent; Sonazoid; liver; VX-2; rabbit

Ultrasonography (US) is useful for diagnosis of hepatic focal lesions, which is based on their distinctive echogenicities; the gray-scale morphologic features. To improve diagnostic ability of detection and characterization of tumors, contrast imaging has been used in diagnostic imaging, e.g. $\mathrm{X}$-ray computed tomography $(\mathrm{CT})$ or magnetic resonance imaging (MRI), but has not yet been popular in US since contrast agents for US are not at clinical satisfaction level. Intravenous US contrast agents available for clinical use or the agents under development are basically microbubbles whose size is comparable to or less than red blood cells. First-generation agent consisted of air-microbubbles, Levovist (SH U 508A; Schering, Berlin, Germany), is commercially available in many countries, ${ }^{1-6)}$ but short imaging duration and weak contrast effect due to instability of the microbubbles could provide only limited diagnostic information. ${ }^{1,2)}$ In order to improve the contrast effects, second-generation agents that use less soluble gases for microbubbles are being developed and now clinically available in limited western countries. ${ }^{1,6-9)}$ Viewpoint from US imaging machine, to detect microbubbles more sensitively, harmonic imaging technology has been developed to visualize the harmonic echo from the microbubbles: backscatter signal (echo) from the microbubbles contains more harmonic component than that from tissues. ${ }^{1)}$

One of the second-generation agents, Sonazoid (previously NC100100, Amersham Health, Oslo, Norway $)^{7,10)}$ has been reported to enhance perfusion of the myocardium and other organs. ${ }^{7,11-14)}$ In the previous study, ${ }^{15)}$ we have characterized contrast enhancement of Sonazoid on the liver of metastatic carcinoma-model (VX-2) in rabbits, ${ }^{16,17)}$ and demonstrated promising contrast effect on the detection and delineation of liver tumors. This is due to uniqueness of Sonazoid whose microbubbles are likely to be taken up by Kupffer cells (liver macrophages) in the healthy liver and enhances contrast of the liver parenchyma during the delayed phase, which usually occurs within $10 \mathrm{~min}$ after the injection. In contrast, tumor that lacks Kupffer cells was not enhanced resulting in clear negative contrast of the tumor.

In the previous study, comparison of the tumor contrast with histopathology of the tumors could not be examined in detail, since resolution of US images that had been acquired using the harmonic imaging technique was not enough to compare with microscopic images. To improve resolution and signal-to-noise ratio of this harmonic technique, phase inversion imaging (PII) technique was then developed and now become available in the commercial US machine. ${ }^{6,18,19)}$ PII can detect harmonic echoes from the microbubble contrast agent with greater sensitivity and without the loss of spatial resolution often seen in the harmonic imaging.

Localization of the microbubbles in the liver Kupffer cells has been demonstrated in rat by using transmission electron microscopy (TEM) ${ }^{20)}$ However, the liver contrast effect in rats, such as dose and contrast duration, have not been fully characterized since rat liver was too small to image using clinical US machine. It is favorable to determine the microbubble distribution in the same species whose liver contrast with Sonazoid has already been characterized.

In this study, we used normal and the rabbits with VX-2 tumor in the liver 1) to demonstrate hepatic tumor detectability of Sonazoid with PII by visual assessment and videodensitometric analysis, 2) to compare the contrast effect and histopathology of the tumor by taking advantage of high resolution of PII, 3) to identify the microbubbles distribution in the rabbit liver at the delayed phase by TEM.

\section{MATERIALS AND METHODS}

Ultrasound Contrast Agent Sonazoid (Amersham Health, Oslo, Norway) is a lipid-stabilised suspension of per- 
fluorobutane microbubbles. The microbubbles have a relatively narrow size-distribution, with a median diameter between 2.4 and $3.5 \mu \mathrm{m}$, and the concentration is approximately $1 \times 10^{9}$ particles $/ \mathrm{ml}$. Over doses inhibit ultrasound penetration in deeper zone of liver and cause contrast attenuation, so that $0.12 \mu \mathrm{l}$ microbubbles $/ \mathrm{kg}$, equal to the recommended clinical dose, was selected through imaging studies.

Implantation of Tumors into Rabbits All procedures using animals were performed in accordance with the institutional guidelines of Daiich Pharmaceutical Co., Ltd. concerning animal experimentation. VX-2 tumor tissue cubes (approximately $2 \times 2 \times 2 \mathrm{~mm}$ ) were harvested from carrier rabbits and implanted into the liver of 6 male New Zealand White rabbits (body mass: $2.5-3.0 \mathrm{~kg}$ ) while the animals were under general anesthesia (pentobarbital and halothane).

Imaging Procedures Imaging was performed 7-11 d after tumor implantation. Animals were anesthetized with $20 \mathrm{mg} / \mathrm{kg}$ (IV) pentobarbital and halothane ( $1-2 \%$ in air) before being secured in a supine position. After the insertion of a tracheal tube, a respirator for experimental animals (SN480-5; Shinano Seisakusyo, Tokyo, Japan) was used to provide each animal with room air. An injection of Sonazoid was given though a 22-gauge catheter inserted into the ear vein. The tubing was flushed with $3 \mathrm{ml}$ of saline just after injection of the contrast agent.

A commercial ultrasound system (Elegra; Siemens, Erlangen, Germany) equipped with an electronic linear probe (7.5L40) was used. With the mechanical ventilation paused temporary to simulate a breath-hold, a continuous scan $(>10$ frames per second) was performed percutaneously at 6.0 MHz for fundamental B-mode imaging (FI) and 3.06.0 MHz for PII. Firstly, before administration of Sonazoid, sweep-scan of the whole liver with FI and PII images were obtained at an acoustic power (PWR) of 100\%. With the PWR setting lowered to $10 \%$ of the maximum in order to avoid microbubble destruction, Sonazoid was administered with the probe fixed by means of a custom-designed clamp to maintain position above the tumors for $30 \mathrm{~s}$ (early phase). Then the imaging was once stopped, and at $10 \mathrm{~min}$ after the administration, the whole liver was sweep-scanned again (delayed phase).

After the imaging study, all animals were sacrificed with an overdose of pentobarbital. Autopsies were performed to confirm the presence of tumors and to provide histopathologic observations regarding the vascularity and the distribution of Kupffer cells for subsequent comparison with ultrasound images.

Imaging Analysis Ultrasound images were continuously recorded onto S-VHS videotapes and a video frame grabber was used to digitize and transfer the images to the image analysis system. An off-line image analysis system (Color Cardiology Work Station; Freeland Systems, Boulder, CO) was used to calculate video signal density. In this system, the gray-scale level ranges from 0 to 255. Appropriate regions of interest (ROIs) included the tumor and healthy liver parenchyma, and the echo density of the ROIs and respective evaluations were taken in each of 3 imaging modes: pre-contrast FI (non-contrast FI), pre-contrast PII (non-contrast PII), or post-contrast PII in the delayed phase (contrast PII). The absolute difference between the ROIs of tumor and healthy parenchyma was designated as the tumor contrast, an index of tumor detectability.

In the VX-2 tumor detectability study, visual assessment was also performed. Each lesion was assessed with 3 readers blinded to treatment retrospectively using randomly chosen videotapes on which the sweep-scanned video images of non-contrast and the delayed phase of Sonazoid were recorded. The readers were asked to assess the existence and position of the tumor in each imaging mode while remaining blind to both implantation status and Sonazoid treatment.

Ultrastructural Analysis Six healthy male New Zealand White rabbits not bearing tumors were intravenously administered Sonazoid $(0.12,1.2$ or $12 \mathrm{ml}$ microbubbles $/ \mathrm{kg}$, equal to 1,10 or 100 times of recommended clinical dose, respectively) or saline as control. Animals were sacrificed with an overdose of pentobarbital at $10 \mathrm{~min}$ after administration, and other animals administered the highest dose were sacrificed at 5 and $30 \mathrm{~min}$ after administration. The liver was excised from each rabbits and cut into $1 \times 1 \times 1 \mathrm{~mm}$ pieces. These samples were fixed in $3 \%(\mathrm{v} / \mathrm{v})$ glutaraldehyde and postfixed in $1 \%(\mathrm{v} / \mathrm{v}) \mathrm{OsO}_{4}$ in $0.1 \mathrm{~mol} / 1$ sodium-phosphate buffer ( $\mathrm{pH}$ 7.4). After dehydration in a graded alcohol series, the samples were embedded in Queto-812 (Nisshin EM, Tokyo), and ultrathin sections were cut, stained with uranyl acetate and lead citrate, and finally examined under transmission electron microscopes (LEM2000; Topcon, Tokyo; or H500; Hitachi, Tokyo). In each animal, approximately 20 to 30 Kupffer cells were examined.

Statistical Analysis All videodensity data are expressed as the mean \pm S.D. Statistical analysis was performed using one-way analysis of variance and Tukey's multiple comparison tests. Differences resulting in a $p<0.05$ were considered significant.

\section{RESULTS}

Visual and Quantitative Analysis Each one small VX2 tumor was confirmed in the liver of each of all 6 animals that had undergone implantation. All tumors were smaller than $12 \mathrm{~mm}$ in diameter $(4-5 \mathrm{~mm} \times 6-12 \mathrm{~mm})$. It was difficult to detect such small tumors with both FI and PII noncontrast imaging modes (Figs. 1A, B). The sensitivity of tumor detection by readers blinded to treatment was $0 \%(0 / 6$, FI) or $17 \%(1 / 6, \mathrm{PII})$ during non-contrast imaging. Conversely, all tumors were detected when PII was used during the delayed phase of Sonazoid treatment (6/6: sensitivity $100 \%)$.

The videodensitometric tumor contrast during the delayed phase was significantly greater than both non-contrast imaging modes $(p<0.05$, Fig. 2). This result correlates closely with the tumor detectability results made by readers blinded to treatment.

Contrast Effects and Histopathology During the early phase, shortly after injection of Sonazoid, the main vessels and tumor rim were enhanced; several seconds later, the entire liver was enhanced (Fig. 1C). Ring enhanced tumor rim, which was enhanced first, corresponded to the border and living tumor area. The necrotic region with no vascularity showed up as a contrast defect (Fig. 1C).

During the delayed phase, while the contrast enhancement of vessels disappeared, the echogenicity of healthy hepatic tissue remained high. Therefore, liver lesions showed up as 
A

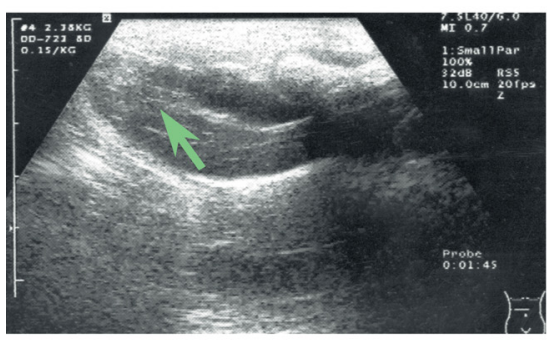

B

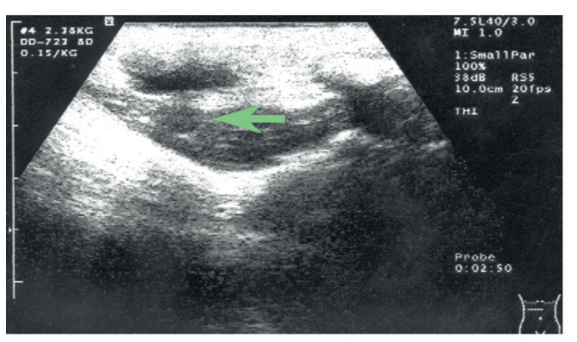

C

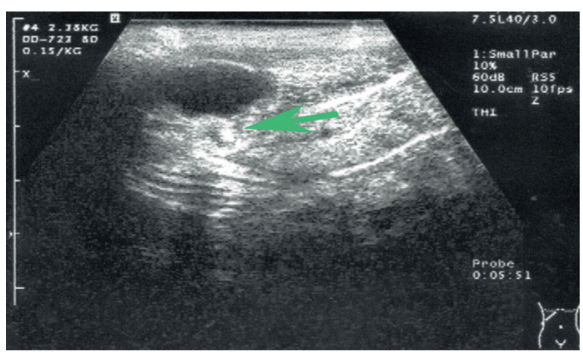

$\mathrm{D}$

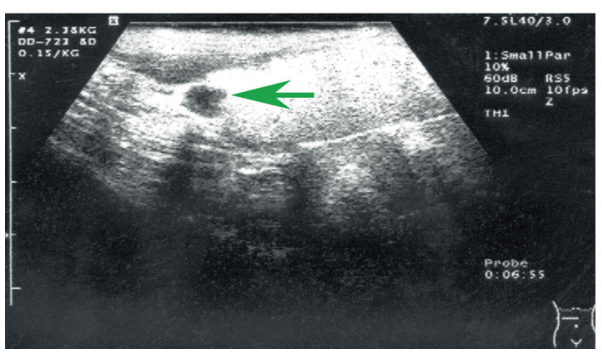

$\mathrm{E}$

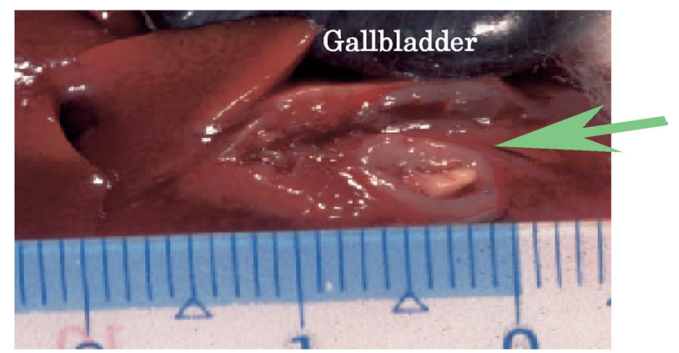

F
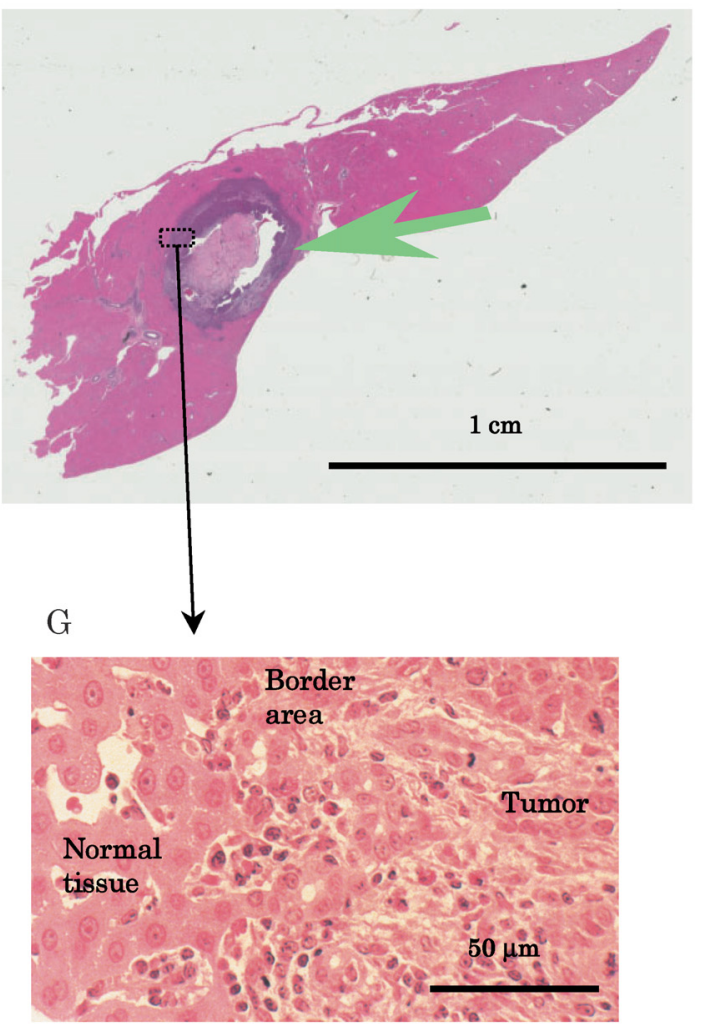

Fig. 1. The Liver of VX-2 Bearing Rabbit Imaged in Fundamental B-mode (A), Phase Inversion Imaging Mode (B-D) before (B), Immediately after (Early Phase, C), and $10 \mathrm{~min}$ after (Delayed Phase, D) Injection of $0.12 \mathrm{ml}$ Microbubbles $/ \mathrm{kg}$ of Sonazoid in Phase Inversion Imaging

(E) Macroscopic and (F) microscopic (HE stain) observations show radiologic-histopathologic correlation. (G) Further magnification for minute observation. Arrows show the location of a VX-2 tumor.

dark spots in a light background. The defect area was larger than that observed during the early phase (Fig. 1D). This defect area included the viable region of tumor surrounding the necrotic region. Subsequent histologic observations (Figs. $1 \mathrm{E}-\mathrm{G}$ ) revealed that: (a) Vascularity was richer in the living region of the tumor and in the border area compared with healthy tissue, whereas the necrotic region was lacking in vascularity; (b) Kupffer cells existed in both healthy tissue and the border area. In contrast, no Kupffer cells were observed in the tumor (Table 1).

Ultrastructural Analysis of the Liver Under a transmission electron microscope with relatively large microscopic field, a few vacuole-like structures were observed in the hepatic Kupffer cells of rabbits administered 10 times the recommended clinical dose of Sonazoid, and more frequently
Table 1. Microscopic Examination of Specimens Removed from VX-2 Bearing Rabbits

\begin{tabular}{lcc}
\hline \hline \multicolumn{1}{c}{ Region } & Kupffer cell $^{a)}$ & Vascularity $^{b)}$ \\
\hline Normal tissue of liver & +++ & + \\
Border area & ++ & ++-+++ \\
Living region of tumor & - & ++-+++ \\
Necrotic region & - & -
\end{tabular}

a) distribution of Kupffer cells was categorized as follows: (-), no Kupffer cell was observed; $(++)$, obviously smaller number of Kupffer cells than that in the normal tissue was observed; $(+++)$, large number of Kupffer cells was observed. $b$ ) Vascularity was categorized with four grade as follows: $(-)$, no vascular was observed; $(+)$, normal vascularity; $(++)$, rich vascularity; $(+++)$, extra rich vascularity.

in that administered 100 times the recommended clinical dose, but no such structures were found in that of rabbits ad- 
ministered saline or the recommended clinical dose (Fig. 3). These vacuole-like structures were round and the size was around $2 \mu \mathrm{m}$ that was comparable to the size of Sonazoid microbubbles. Not only one but also several of such structures were found in a Kupffer cells. The structure was found in the Kupffer cells from $5 \mathrm{~min}$ to $30 \mathrm{~min}$ after administration of the highest dose of Sonazoid. Except the vacuole-like structures in Kupffer cells, no difference was observed between control and Sonazoid administered animals.

\section{DISCUSSION}

In this study, firstly we examined the detectability of small VX-2 tumors in the rabbit liver with Sonazoid coupling with PII. Blinded readers should detect the tumor on video images

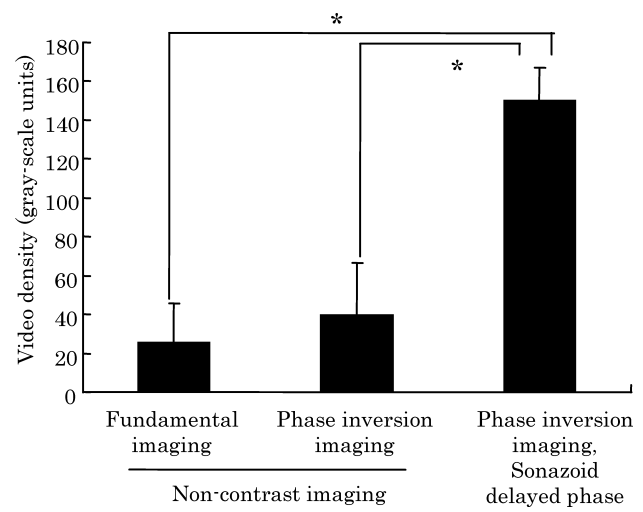

Fig. 2. Contrast of Videodensitometric Measurements of Tumor and Healthy Tissues in the Liver after Injection of Sonazoid $(0.12 \mu 1$ Microbubbles $/ \mathrm{kg}$ ) into Rabbits (Delayed Phase)

The columns, left to right, show results for the non-contrast fundamental B-mode, non-contrast phase-inversion imaging mode, and contrast phase-inversion imaging mode. * Significantly differences compared with the values for modes before Sonazoid was administered at $p<0.05$ (Tukey's test).

a

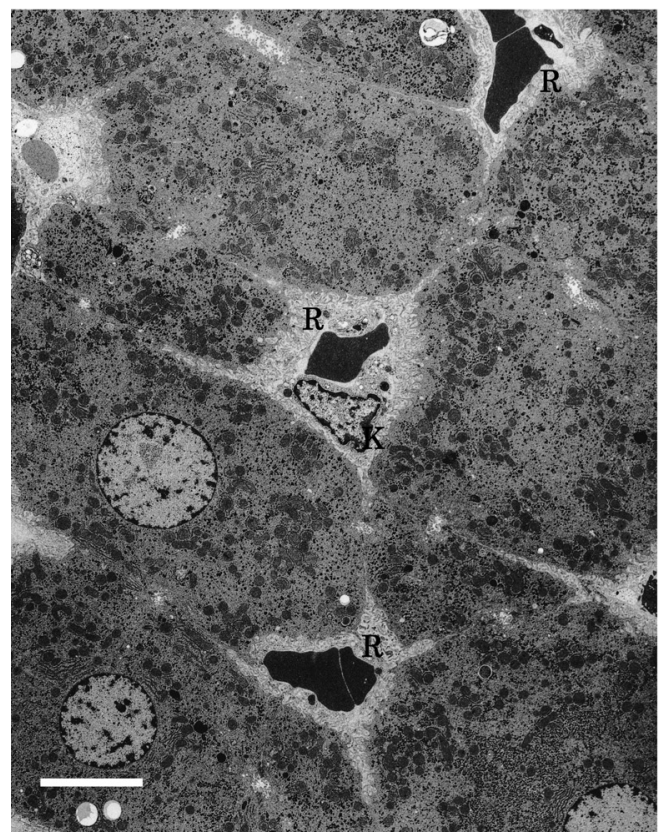

where whole liver was sweep-scanned, while in the previous study using FI and conventional second-harmonic imaging, ${ }^{15}$ the readers only detected the tumor in a fixed image plane that contained tumor. Since it was more challenging to detect tumors not in the plane but in the space of whole liver, more clear delineation and higher contrast of the tumor was required: this is more likely to clinical setting where detection of tumors in real time sweep image is quite important.

Readers detected no tumor in all 6 rabbits bearing VX-2 in the liver in FI, while only one in 6 rabbits in PII without Sonazoid. PII was initially developed for detection and visualization of the harmonic signal specifically reflected from microbubbles, ${ }^{18)}$ however, PII without contrast gradually becomes popular as same as usual FI since clutter noise often seen in FI is suppressed in PII, ${ }^{19)}$ even though harmonic signal from tissue is smaller than fundamental signal. This might be the reason that non-contrast PII could detect one tumor while FI could not. But in a large sense, risk of missing such small tumor may still exist in clinical setting when contrast is not used. Complete detection of tumors during delayed phase of Sonazoid with PII in this study indicates advantage of contrast US with Sonazoid; risk of missing tumors could possibly be reduced and detection of new lesion is expected in clinical setting.

The videodensitometric measurements of tumor contrast also supported the result of higher tumor detectability of Sonazoid with PII. Since tumor contrast was small in both non-contrast images, the readers detected the tumor by distinguishing difference of texture between tumor and healthy liver parenchyma rather than by detecting the contrast between them. In case of delayed phase Sonazoid, the readers detected the clear contrast of tumor than the texture difference. This is quite reasonable since detection of clear contrast is easier than distinguish texture in the video images (animation) where imaging plane changes over frame by

$\mathrm{b}$

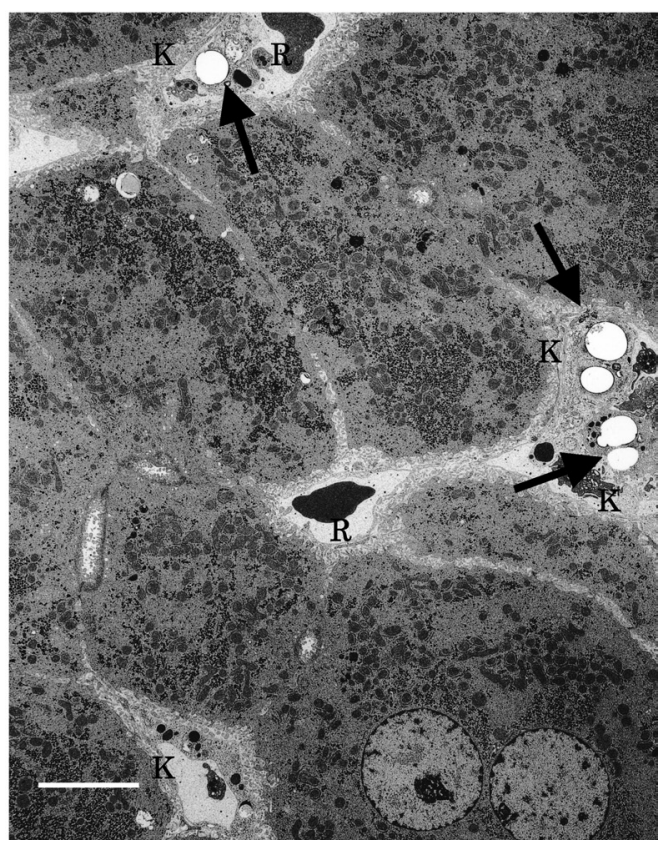

Fig. 3. Transmission Electron Microscopy of the Rabbit Liver Removed 10 min after Administrated Saline (Control) (a) or Sonazoid (12 $\mu 1$ Microbubbles $/ \mathrm{kg}$ ) (b)

One or 2 microbubble-like round-shape spaces in each Kupffer cells (arrows) after administration of Sonazoid. K, Kupffer cell; R, red blood cell. Scale bar=5 $\mu$ m. 
frame to cover whole the liver. In the previous study, all tumors were also detected by difference in echogenicity between lesion and normal tissue using conventional secondharmonic imaging. ${ }^{15)}$ However, the tumor contrast in conventional second-harmonic imaging was not so high as that in PII used in this study. Smaller the tumor size, advantage of clear tumor contrast in delayed phase of Sonazoid with PII may become more conspicuous.

Secondary, we compared dynamics of tumor contrast by Sonazoid with histopathology of VX-2 tumor. Although the tumor cross-section between US image and histopathology was not strictly the same, we compared the both since tumors in all 6 rabbits were mostly globular shape. The histopathologic observation that was rich vascularity in living part of the tumor and necrotic region without vascularity in the center revealed typical feature of VX-2 tumor. ${ }^{21)}$ Ring enhancement of tumor rim in the early phase of Sonazoid with PII well agreed with rich vascularity of the tumor in histopathologic observation. This is quite important in clinical settings, since visualization of features of tumor vasculature is essential for characterization of focal lesion ${ }^{22,23)}$ and this result demonstrated the potential of Sonazoid to visualize the tumor vasculature. In the delayed phase of Sonazoid, healthy parenchyma was enhanced but whole tumor including both living and necrotic part was not enhanced. Since this region was Kupffer cell free part confirmed by histopathologic observation, consistency of US contrast image with histopathology was also confirmed in this phase.

Contrast effect of any contrast agent is greatly influenced by matching with imaging techniques. PII technique largely overcomes the contrast detectability and imaging resolution trade-off by means of summing the responses from inverted transmitting ultrasound waves. .,18,19) $^{2}$ Conventional secondharmonic imaging restricts the received bandwidth and degrades imaging resolution because a harmonic filter is needed to cut off overlapping frequencies of received sound reflected from both the agent and tissue. ${ }^{6)}$ However, in PII, linear echoes from tissue are inverted copies of each other and add up to zero, while the distorted and non-linear components of microbubble echoes reinforce each other when summed and produce a strong harmonic signal. Therefore, the phase-inversion technique overcomes the conflict between the requirements of contrast and resolution in harmonic imaging and provides greater sensitivity, thus allowing low incident power and non-destructive, high-contrast imaging of microbubble-containing tissues and fluids. ${ }^{6,19)}$ Because of high spatial and temporal resolution, exact radiologic and histopathologic comparison could be performed. The results of this comparison confirmed that Sonazoid accumulated in healthy hepatic parenchyma after its elimination from the blood stream. A first-generation agent, Levovist, also has a liver-specific phase. ${ }^{1,3,5)}$ They soon disappear, however, because the microbubbles are easy to be destroyed even when an area is scanned with US only once. ${ }^{3)}$ In this study, Sonazoid used with PII provided strong and long-lasting contrast enhancement that resulted in excellent tumor detection at low acoustic power. A low-power setting will prolong the duration of the contrast effect and enable more detailed inspections. $\left.{ }^{6}\right)$ Thus, Sonazoid might be useful in thorough searches for minute liver metastases, without the need for any additional contrast agent.
In addition to imaging analysis, TEM results from rabbit tissue indicated uptake of intact microbubbles by Kupffer cells more directly. As the Kupffer cells, the liver macrophages whose major biological function is phagocytosis, are present in large numbers in the liver and directly exposed to blood stream, ${ }^{24)}$ microbubbles of the size of Sonazoid could be entirely phagocytosed by Kupffer cells. ${ }^{25,26)}$ Therefore, it was convinced that vacuole-like structure observed in the Kupffer cells was microbubbles of Sonazoid. This is quite reasonable since the structure was round shape, similar size to Sonazoid microbubbles, non-electron dense, and found more frequently at the highest dose. Similar structure was also observed in Kupffer cells in rats after intravenous administration of Sonazoid ${ }^{20)}$ and activated leukocytes incubated with another lipid microbubbles. ${ }^{27)}$ Although visual contrast enhancement of the liver parenchyma was observed at the recommended clinical dose, no such structure was found in the TEM. This indicated that all the Kupffer cells were not needed to take up the microbubbles to obtain delayed phase contrast effect and the result was quite coincided with the one from rat study. Kindberg et al. could find only 5 vacuole-like structures in 67 Kupffer cells in the liver from total of 4 rats at dose of 60 times the recommended clinical dose. ${ }^{20)}$ Moreover, sampling time period of this ultrastructual analysis, 5 to $30 \mathrm{~min}$ after administration, is within the time frame of delayed phase investigated in our previous study. ${ }^{15)}$ These results also supported that these vacuole-like structures in Kupffer cells were Sonazoid microbubbles and they provided the contrast effect on the liver parenchyma.

In conclusion, Sonazoid used with PII greatly improved the conspicuity and detectability of small VX-2 tumors. As confirmed by radiologic and histopathologic observations, the contrast enhancement observed during the early phase highlighted the vascurarity, especially the neovascularity, of tumors. Conversely, the contrast enhancement during the delayed phase is thought to reflect the distribution of Kupffer cells. In addition, ultrastructural observations supported that Sonazoid microbubbles were taken up by Kupffer cells. Based on these results, the early phase can be called vascular-specific imaging, and the delayed phase can be called parenchyma-specific, especially Kupffer cell-specific imaging. Sonazoid with PII promises to be a useful technique for detecting tumors in the liver by visualization of tumor neovascularity during the early phase and of dark contrast defects during the delayed phase.

\section{REFERENCES}

1) Calliada F., Campani R., Bottinelli O., Bozzini A., Sommaruga M. G., Eur. J. Radiol., 27, S157-160 (1998).

2) Kim T. K., Han J. K., Kim A. Y., Choi B. I., J. Ultrasound Med., 18, 737-743 (1999).

3) Heckemann R. A., Cosgrove D. O., Blomley M. J. K., Eckersley R. J., Harvey C. J., Mine Y., Radiology, 216, 592-596 (2000).

4) Runge V. M., Invest. Radiol., 2, 123-130 (2001).

5) Suzuki S., Iijima H., Moriyasu F., Sasaki S., Yanagisawa K., Miyahara T., Oguma K., Yoshida M., Horibe T., Ito N., Kakizaki D., Abe K., Tsuchiya K., Hepatol. Res., 29, 122-126 (2004).

6) Frinking P. J. A., Bouakaz A., Kirkhorn J., Cate F. J. T., de Jong N., Ultrasound Med. Biol., 26, 965-975 (2000).

7) Marwick T. H., Brunken R., Meland N., Brochet E., Baer F. M., Biner T., Flachskampf F., Kamp O., Nienaber C., Nihoyannopoulos P., Pierard L., Vanoverschelde J., Wouw P., Lindvall K., J. Am. Coll. Cardiol., 32, $1260-1269$ (1998) 
8) Wilson S. R., Burns P. N., Muradali D., Wilson J. A., Lai X., Radiology, 215, 153-161 (2000).

9) Wei K., Crouse L., Weiss J., Villanueva F., Schiller N. B., Naqvi T. Z., Siegel R., Monaghan M., Goldman J., Aggarwal P., Feigenbaum H., DeMaria A., Am. J. Cardiol., 91, 1293-1298 (2003).

10) Sontum P. C., Ostensen J., Dyrstad K., Hoff L., Invest. Radiol., 34, 268-275 (1999).

11) Marelli C., Am. J. Cardiol., 86, 10G-13G (2000).

12) Yao J., Teupe C., Takeuchi M., Avelar E., Sheahan M., Connolly R., Ostensen J., Pandian N. G., J. Am. Soc. Echocardiogr., 13, 570-581 (2000).

13) Hagen E. K., Forsberg F., Aksnes A., Merton D. A., Liu J., Tornes A., Johnson D., Goldberg B. B., Invest. Radiol., 35, 118-124 (2000).

14) Hagen E. K., Magnusson A., Aksnes A. K., Norberg M., Acta Radiol., 42, 225-229 (2001).

15) Watanabe R., Matsumura M., Chen C. J., Kaneda Y., Ishihara M., Fujimaki M., Biol. Pharm. Bull., 26, $1272-1277$ (2003).

16) Phillips J. J., Chang S. L., Vargas H. I., Dickman P. S., Butler J. A., Lipcamon J. D., Magn. Reson. Imaging, 9, 201-204 (1991).

17) Maruyama H., Matsutani S., Saisho H., Kamiyama N., Yuki H., Miyata K., Ultrasound Med. Biol., 26, 1429-1438 (2000).

18) Simpson D. H., Chin C. T., Burns P. N., IEEE Trans. Ultrason. Ferr.
Freq. Cont., 46, 372-382 (1999).

19) Burns P. N., Wilson S. R., Simpson D. H., Invest. Radiol., 35, 58-71 (2000).

20) Kindberg G. M., Tolleshaug H., Roos N., Skotland T., Cell. Tissue Res., 312, 49-54 (2003).

21) Kono Y., Moriyasu F., Nada T., Suginoshita Y., Matsumura T., Kobayashi K., Nakamura T., Chiba T., Ultrasound Med. Biol., 23, $719-726$ (1997).

22) Matsui O., Kadoya M., Kameyama T., Yoshikawa J., Takashima T., Nakanuma Y., Unoura M., Kobayashi K., Izumi R., Ida M., Kitagawa K., Radiology, 178, 493-497 (1991).

23) Kudo M., Hepatogastroenterology, 45, 1226-1231 (1998).

24) Wake K., Decker K., Kirn A., Knook D. L., McCuskey R. S., Bouwens L., Wisse E., Int. Rev. Cytol., 118, 173-229 (1989).

25) Rahman Y. E., Cerny E. A., Patel K. R., Lau E. H., Wright B. J., Life Sci., 31, 2061-2071 (1982).

26) Ogawara K., Yoshida M., Higaki K., Kimura T., Shiraishi K., Nishikawa M., Takakura Y., Hashida M., J. Controlled Release, 59, $15-22$ (1999).

27) Lindner J. R., Dayton P. A., Coggins M. P., Ley K., Song J., Ferrara K., Kaul S., Circulation, 102, 531-538 (2000). 